\title{
1 Algebraic theorem of selection by spatial sorting
}

2

$3 \quad$ Nikunj Goel

4

5 Department of Integrative Biology, the University of Texas at Austin, Austin, TX, USA, 78712

6 email: nikunj.goel@utexas.edu

7

8 Competing Interest Statement: I declare no competing interest.

9 Keywords: Spatial sorting, sorting theorem, natural selection, Price's theorem

10 This file includes: Main Text, Figure 1, and Table 1 


\section{Abstract}

12 Heritable variation in traits that enhance dispersal rates can accumulate at population margins by

13 spatial sorting. This mechanism of selection differs from natural selection as evolutionary change

14 can ensue even in the absence of differential lifetime reproductive success. Although evidence

15 suggests that populations are rapidly evolving at margins due to global change pressures, such as

16 invasions and range shifts, we lack a mathematical theory of spatial sorting to understand these

17 evolutionary patterns. To this end, we present an algebraic theorem, or the sorting theorem, to

18 elucidate the nature of selection at margins, which can, in turn, facilitate axiomatic development

19 of spatial sorting theory. The role of the sorting theorem in guiding research in this context is

20 analogous to that of Price's theorem in natural selection theory. 


\section{Introduction}

Heritable variation in traits that confer differential lifetime reproductive success can drive

24 evolutionary change by natural selection (1). However, at population margins, e.g., invasion fronts

25 (2) and metapopulations (3), evolutionary change can ensue even in the absence of differential

26 lifetime reproductive success (4). This mechanism of biological selection, or spatial sorting, relies

27 on non-random gene flow that segregates traits at margins based on differential dispersal rates (5).

28 Individuals with traits that confer dispersal advantage accumulate at margins, and subsequent

29 assortative mating between these individuals yields evolutionary change towards traits with increased dispersal rates (4).

There is strong evidence that natural populations have abundant heritable variation in dispersal traits (6) and often experience spatial sorting (7). Yet, unlike natural selection theory, we do not know the exact biological conditions that drive selection at margins, and we lack a comprehensive mathematical theory of selection by spatial sorting (4). This task of building spatial sorting theory will likely gain prominence over the coming decades due to increasing global change pressures at margins and yield new insights into how spatial biogeographic patterns are maintained.

The traditional approach to constructing a model in evolutionary theory starts with making simplifying assumptions for a given biological context, followed by mathematical analysis to arrive at analytical predictions. Indeed, recent work on spatial sorting has taken this approach to build models $(8,9)$. However, these special case models inevitably overshadow the underlying

42 general principles of selection. Some argue an alternative approach to building selection theory

43 (10) - one first defines unambiguous principles based on a minimalistic set of assumptions that

44 applies to a large class of biological systems of interest. Theorems that arise from the analysis of 45 these principles form the mathematical foundations of the selection theory from which special case models can be derived and studied. Building selection theory this way provides an axiomatic guide to build and criticize models.

Price's algebraic theorem has served this purpose in building and interpreting natural selection theory (11). However, Price's theorem is inept at describing spatial sorting, as the theorem fundamentally differs in how evolutionary change is quantified. Price's theorem

51 calculates evolutionary change by natural selection over a spatially static frame of reference. In 
52 contrast, spatial sorting is an evolutionary process where trait changes are observed over a moving

53 frame of reference such that the population margin is stationary with respect to the observer (8).

54 To address this gap, we present the algebraic theorem of spatial sorting, which we call the

55 sorting theorem. The sorting theorem elucidates $(i)$ the biological conditions that drive

56 evolutionary change at population margin and (ii) allow axiomatic model building and criticism.

57 (iii) Using the sorting theorem, we derive standard population and quantitative genetic models of

58 spatial sorting, which can allow us to measure and interpret selection patterns in natural

59 populations.

\section{Results}

62 The Sorting Theorem. Consider two patches $x$ and $x^{\prime}$ (Fig. 1A). A population of $N$ 63 ancestors occupy patch $x$ at time $t$. The patch $x^{\prime}$ is empty. Ancestor $i$ has a trait value $\phi_{i}$, which 64 can be any numerically measurable property, e.g., a polygenic trait or frequency of an allele in a 65 genotype or breeding values. At a later time $t^{\prime}$, some descendants occupy $x^{\prime}$. The trait value of the $66 j^{\text {th }}$ descendant $\left(j=1,2, \ldots, W_{i}\right)$ is $\delta_{i, j}$ more than the trait value of its ancestor, $i$. We track the

67 position of descendants using an indicator function $\mathbf{1}_{(i, j) \in x^{\prime}}$, which is one if the $j^{\text {th }}$ descendant of $68 i$ is in $x^{\prime}$, and zero otherwise. Based on this notation, the mean trait value of descendants in $x^{\prime}$ is

$$
\frac{\sum_{i=1}^{N} \sum_{j=1}^{W_{i}} \mathbf{1}_{(i, j) \in x^{\prime}}\left(\phi_{i}+\delta_{i, j}\right)}{\sum_{i=1}^{N} \sum_{j=1}^{W_{i}} \mathbf{1}_{(i, j) \in x^{\prime}}}
$$

69 The numerator is the sum of trait values of all descendants in $x^{\prime}$ by sequentially adding trait values

70 of descendants of a given ancestor, for all ancestors. The denominator is the number of descendants 71 in $x^{\prime}$.

72 We define $V_{i}$ as the fraction of $W_{i}$ descendants of $i$ in $x^{\prime}$ and $\bar{\delta}_{i}$ as the difference between 73 the mean trait value of $i$ 's descendants in patch $x^{\prime}$ and $i$ 's trait value. By rearranging terms in 1 , 74 we show the evolutionary change $(\Delta \bar{\phi})$ at population margin, i.e., the difference between the mean 75 of trait values of descendants in patch $x^{\prime}$ at time $t^{\prime}$ and ancestors in patch $x$ at time $t$, is

$$
\Delta \bar{\phi}=\frac{1}{\overline{V W}}[\mathbb{C} \mathbb{N}(V W, \phi)+\mathbb{E}(V W \bar{\delta})]
$$

76 where 


$$
V_{i} W_{i}=\sum_{j=1}^{W_{i}} \mathbf{1}_{(i, j) \in x^{\prime}}
$$

77 is the number of descendants of ancestor $i$ in patch $x^{\prime}$. We refer to $V_{i} W_{i}$ and $V_{i} W_{i} / \overline{V W}$ as the 78 sorting fitness and relative sorting fitness of $i$, respectively (see SI for derivation). Accordingly, $79 \overline{V W}=\mathbb{E}(V W)$ is the mean sorting fitness of the ancestors.

The sorting theorem (Eq. 2) partitions evolutionary change into two terms. The $\mathbb{C O V}(V W, \phi) / \overline{V W}$ term is the evolutionary change resulting from differential dispersal,

82 reproduction, and survival. This term is an abstraction of selection. Note that directional evolutionary change can occur at margins even in the absence of differential lifetime reproductive success, a key requisite for natural selection. The $\mathbb{E}(V W \bar{\delta}) / \overline{V W}$ term captures transmission bias 85 between ancestors and descendants. This bias arises from biological processes during reproduction, e.g., mutation, recombination, and regression towards the mean. Note the conspicuous similarity in the mathematical structure of Price's theorem and the sorting theorem.

On the one hand, juxtaposing Price's theorem and the sorting theorem suggest that even though these theorems measure the evolutionary change in different ways, mathematics of biological selection is general (12). On the other hand, this juxtaposition elucidates the key difference between the two modes of selection. Unlike natural selection, where the fitness is the number of descendants an ancestor leaves in the next generation, in spatial sorting, the sorting fitness (Eq. 3) is the number of descendants that an ancestor leaves at the population margin, i.e., previously empty patch. This suggests neither differential dispersal ability nor differential lifetime reproductive success are necessary or sufficient to drive spatial sorting. Instead, what ultimately drives selection is heritable variation in traits that confer differential sorting fitness.

Building models of spatial sorting. The sorting theorem also provides a helpful analogy to build selection models of spatial sorting. For any margin population undergoing evolution, one can conceive an imaginary closed system where Price's theorem would yield the same evolutionary change (Fig. 1B) if we assume that descendants in patch $x$ (Fig. 1A) are removed

102 before the census in the closed population (Fig. 1B). The usefulness of this analogy can be shown 103 by noting that we can derive standard population (selection at one locus in haploids and diploids) 104 and quantitative (single and multiple phenotypic traits) genetic models of spatial sorting using the 
105

106

107

108

109

sorting theorem by following methods used to derive models of natural selection from Price's theorem (10) (see Table 1 and SI). Some of these models have been independently derived by others $(8,9)$, which also explains why these models are considered as spatial analogs of natural selection models.

\section{Discussion}

Can we use the sorting theorem to guide the theoretical understanding of evolutionary change at margins? The history of evolutionary research tells us we can. Price's theorem has played a central role in developing natural selection theory by providing new insight into old problems, identifying hidden connections, resolving debates, and clarifying elusive concepts (13). Certainly, the sorting theorem can serve that purpose and then do more. For one, when Price proposed the algebraic theorem of natural selection (11), the mathematics behind many core ideas in natural selection theory had already been laid out (10). This is not the case for spatial sorting, for which much of the theory is yet to be developed $(4,8)$.

Like Price's theorem, the sorting theorem can school our intuition to reason about complex ideas $(11,13)$ and, thereby, guide axiomatic model building and criticism in a given biological context (10). However, establishing the biological context is outside the scope of the sorting theorem. That task is up to the researcher by answering questions such as-what are the entities under selection? How are the traits of interest linked to the sorting fitness of the ancestors? What is the inheritance mechanism? In unison with the biological context, the sorting theorem provides a unified approach to build the selection theory of spatial sorting.

\section{Acknowledgments}

I acknowledge the support from the Stengl-Wyer scholars program. I thank Stephen Stearns, Arun Chavan, Brendan Allison, Tim Keitt, Vagheesh Narasimhan, Athmanathan Senthilnathan, Ben Phillips, Erik Iverson, and Damla Cinoglu for their feedback. 


\section{References}

132 1. C. Darwin, A. Wallace, On the tendency of species to form varieties, and on the perpetuation of varieties and species by natural means of selection. Journal of the Linnean

Society of London 3, 45 - 62 (1858)

135

2. B. L. Phillips, G. P. Brown, J. K. Webb, R. Shine, Invasion and the evolution of speed in toads. Nature 439, 803 (2006).

3. I. Hanski, C. Era lahti, M. Kankare, O. Ovaskainen, H. Sire' n, Variation in migration propensity among individuals maintained by landscape structure Ecology Letters 7, 958-966 (2004).

4. R. Shine, G. P. Brown, B. L. Phillips, An evolutionary process that assembles phenotypes through space rather than through time. Proceedings of the National Academy of Sciences

143 5. P. Edelaar, D. I. Bolnick, Non-random gene flow: an underappreciated force in evolution 144 and ecology. Trends in ecology \& evolution 27, 659--665 (2012).

145 6. M. Saastamoinen et al., Genetics of dispersal. Biological Reviews 93, 574--599 (2018).

146 7. A. Chuang, C. R. Peterson, Expanding population edges: theories, traits, and trade-offs. $147 \quad$ Global Change Biology 22, 494-512 (2016).

148 8. B. L. Phillips, T. A. Perkins, Spatial sorting as the spatial analogue of natural selection. Theoretical Ecology 12, 155--163 (2019).

1509 9. S. Peischl, K. J. Gilbert, Evolution of dispersal can rescue populations from expansion 151 load. The American Naturalist 195, 349--360 (2020).

152 10. S. H. Rice, Evolutionary theory: mathematical and conceptual foundations (Sinauer 153 Associates, MA, 2004).

154 11. G. R. Price, Selection and covariance. Natue 227, 520--521 (1970).

155 12. G. R. Price, The Nature of Selection. Journal of theoretical biology 175, 389--396 (1995).

156 13. S. A. Frank, Natural selection. IV. The Price equation. Journal of Evolutionary Biology 25, 157 1002-1019(2012). 


\section{A}

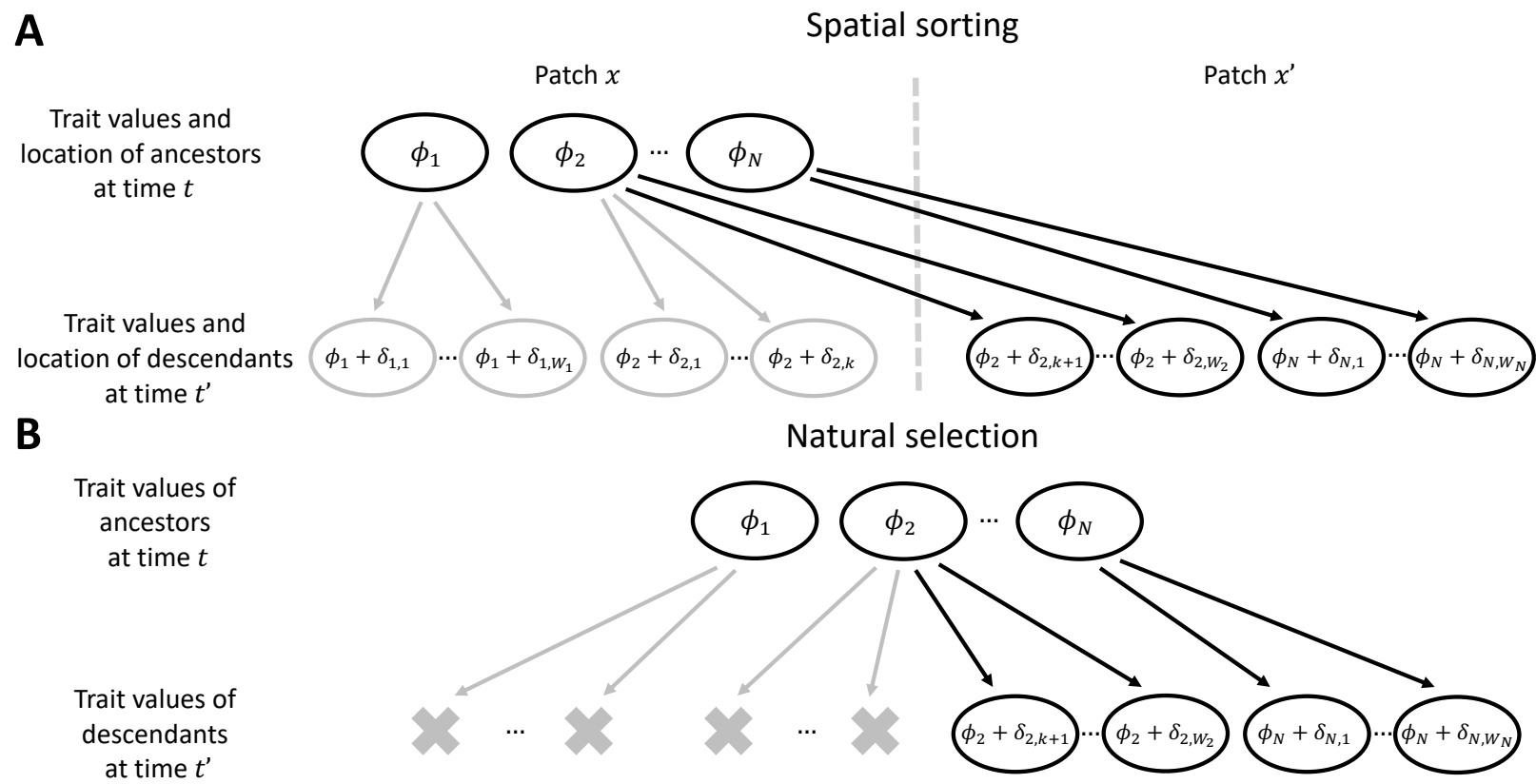

Patch $x$

Spatial sorting at time $t^{\prime}$

Figure 1: (A) Tracking the position and trait values of ancestors and their descendants at the 161 population margin. In this example, the sorting fitness of the $1^{s t}, 2^{n d}$, and $N^{t h}$ ancestor is $0, W_{2}-$ $162 k$, and $W_{N}$, respectively. (B) Constructing a closed population, for which Price's theorem yields 163 the same evolutionary change as the sorting theorem in plot $\mathbf{A}$. 
164 Table 1: Population and quantitative genetic models of spatial sorting (see SI for derivations).

\begin{tabular}{|c|c|c|c|c|}
\hline $\begin{array}{c}\text { Biological } \\
\text { context }\end{array}$ & $\phi_{i}=$ & Evolutionary change & Notation & Ref \\
\hline $\begin{array}{l}\text { Haploids with } \\
\text { genotypes } A \\
\text { and } a\end{array}$ & $\begin{cases}1 & A \\
0 & a\end{cases}$ & $\Delta p=p q \frac{V_{A} W_{A}-V_{a} W_{a}}{\overline{V W}}$ & $\begin{array}{c}p: \text { freq. of allele } A \\
q=1-p: \text { freq. of allele } a \\
V_{A} W_{A}: \text { sorting fitness of allele } A\end{array}$ & $(8,9)$ \\
\hline $\begin{array}{l}\text { Diploids with } \\
\text { genotypes } A A \text {, } \\
A a, \text { and } a a\end{array}$ & $\left\{\begin{array}{cc}1 & A A \\
0.5 & A a \\
0 & a a\end{array}\right.$ & $\Delta p=p q \frac{V_{A} W_{A}^{*}-V_{a} W_{a}^{*}}{\overline{V W}}$ & $\begin{array}{c}V_{A} W_{A}^{*}: \text { marginal sorting fitness } \\
\text { of allele } A\end{array}$ & (8) \\
\hline $\begin{array}{l}\text { Single } \\
\text { phenotypic } \\
\text { trait }\end{array}$ & $\begin{array}{c}\text { A scalar } \\
\text { phenotypic } \\
\text { trait }\end{array}$ & $\Delta \bar{\phi}=\frac{1}{\overline{V W}} G \beta_{V W, \phi}$ & $\begin{array}{c}\beta_{V W, \phi}: \text { regression of sorting } \\
\text { fitness on the trait } \\
G \text { : additive genetic variance }\end{array}$ & - \\
\hline $\begin{array}{l}\text { Multiple } \\
\text { phenotypic } \\
\text { traits }\end{array}$ & $\begin{array}{l}\text { A vector of } \\
\text { phenotypic } \\
\text { traits }\end{array}$ & $\Delta \overrightarrow{\bar{\phi}}=\frac{1}{\overline{V W}} \boldsymbol{G} \vec{\beta}_{V W, \phi \mid}$ & $\begin{array}{l}\vec{\beta}_{V W, \phi \mid}: \text { a vector of partial } \\
\text { regression of sorting fitness on } \\
\text { each of the traits. } \\
G \text { : Genetic covariance matrix }\end{array}$ & - \\
\hline
\end{tabular}

